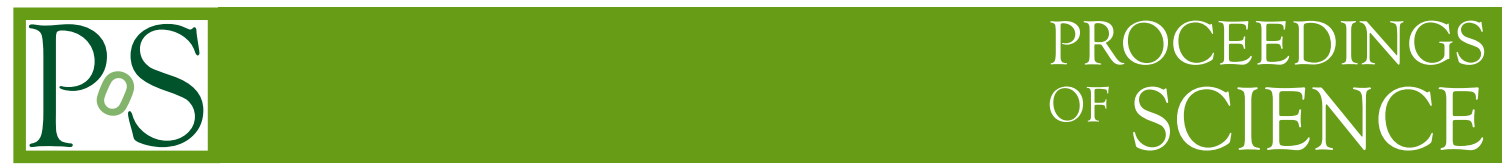

\title{
Parton branching TMD method and multi-jet production
}

\author{
Armando Bermudez Martinez $^{a, *}$ and Francesco Hautmann ${ }^{b, c}$ \\ ${ }^{a}$ Deutsches Elektronen-Synchrotron, D-22607 Hamburg, FRG \\ ${ }^{b}$ RAL, Chilton OX11 OQX and University of Oxford, OX1 3NP \\ ${ }^{c}$ Elementaire Deeltjes Fysica, Universiteit Antwerpen, B 2020 Antwerpen, Belgium \\ E-mail: armando.bermudez.martinez@desy.de, \\ francesco.hautmann@physics.ox.ac.uk, armando.bermudez.martinez@desy.de, \\ francesco.hautmann@physics.ox.ac.uk
}

Transverse momentum dependent (TMD) parton distributions obtained from the Parton Branching (PB) method are combined with next-to-leading-order (NLO) calculations of jet production to obtain predictions for LHC jet final states. In addition, a new initial state Parton Shower, which is based on the TMD distributions, and final state Parton Showers are included together with hadronization. We compare our predictions with jet and $\mathrm{Z}+$ jet measurements performed at the LHC, finding good agreement. We present results indicating the need for multi-jet merging with PB-TMDs.

40th International Conference on High Energy physics - ICHEP2020

July 28 - August 6, 2020

Prague, Czech Republic (virtual meeting)

\footnotetext{
${ }^{*}$ Speaker
} 


\section{Introduction}

While advanced formalisms have been developed which treat the effects of transverse momentum dependent (TMD) parton distributions in inclusive and semi-inclusive observables [1], TMD effects on exclusive final states with high jet multiplicity remain largely unexplored.

In this report we obtain results which point to the need for incorporating simultaneously the contribution of different jet multiplicities in the final state and the contribution of initial-state TMD evolution. For these studies we make use of the parton branching (PB) formulation of TMD evolution set out in [2].

\section{PB approach to TMD evolution}

The PB method [2] uses the unitarity picture of parton evolution [3, 4], commonly used in showering algorithms, for both collinear and TMD parton distributions. In this approach, the concept of resolvable and non-resolvable branchings is applied by using Sudakov form factors. A soft-gluon resolution scale $z_{M}$ [5] is introduced to separate resolvable and non-resolvable branchings. The Sudakov form factors, which describe the evolution without resolvable branching from one scale $\mu_{0}$ to another scale $\mu$, are given in terms of the resolvable splitting probabilities $P_{b a}^{(R)}\left(\alpha_{s}, z\right)$ as follows,

$$
\Delta_{a}\left(\mu^{2}, \mu_{0}^{2}\right)=\exp \left[-\sum_{b} \int_{\mu_{0}^{2}}^{\mu^{2}} \frac{\mathrm{d} \mu^{\prime 2}}{\mu^{\prime 2}} \int_{0}^{z_{M}} \mathrm{~d} z z P_{b a}^{(R)}\left(z, \alpha_{s}\right)\right],
$$

where $a, b$ are flavor indices, $\alpha_{s}$ is the strong coupling, $z$ is the longitudinal momentum splitting variable, and $z_{M}<1$ is the soft-gluon resolution parameter.

The TMD distributions $\mathcal{A}_{a}\left(x, k_{\mathrm{T}}^{2}, \mu^{2}\right)$ fulfill PB evolution equations [2] in terms of evolution kernels $\mathcal{K}_{b a}$, computable in terms of Sudakov form factors, resolvable splitting probabilities and appropriate phase-space constraints. If these constraints are taken of the form prescribed by angular ordering [3, 6, 7], it is shown in [8] that the PB evolution equation returns, once it is integrated over transverse momenta, the coherent-branching equation of [7]. The PB equations can be implemented in a Monte Carlo generator as described in [9]. Using non-perturbative starting distributions $\mathcal{A}_{0, b}\left(x^{\prime}, k_{\mathrm{T}, 0}^{2}, \mu_{0}^{2}\right)$ convoluted with perturbative evolution kernels $\mathcal{K}_{b a}$, we have

$$
\begin{aligned}
x \mathcal{A}_{a}\left(x, k_{\mathrm{T}}^{2}, \mu^{2}\right) & =x \int d x^{\prime} \int d x^{\prime \prime} \mathcal{A}_{0, b}\left(x^{\prime}, k_{\mathrm{T}, 0}^{2}, \mu_{0}^{2}\right) \mathcal{K}_{b a}\left(x^{\prime \prime}, k_{\mathrm{T}, 0}^{2}, k_{\mathrm{T}}^{2}, \mu_{0}^{2}, \mu^{2}\right) \delta\left(x^{\prime} x^{\prime \prime}-x\right) \\
& =\int d x^{\prime} \mathcal{A}_{0, b}\left(x^{\prime}, k_{\mathrm{T}, 0}^{2}, \mu_{0}^{2}\right) \frac{x}{x^{\prime}} \mathcal{K}_{b a}\left(\frac{x}{x^{\prime}}, k_{\mathrm{T}, 0}^{2}, k_{\mathrm{T}}^{2}, \mu_{0}^{2}, \mu^{2}\right) .
\end{aligned}
$$

In the calculations of [9] we use, for simplicity, a factorized form for the starting distribution $\mathcal{A}_{0}$ (in general, the $k_{\mathrm{T}, 0}$ distribution can be also flavor and $x$-dependent),

$$
\mathcal{A}_{0, b}\left(x, k_{\mathrm{T}, 0}^{2}, \mu_{0}^{2}\right)=f_{0, b}\left(x, \mu_{0}^{2}\right) \exp \left(-\left|k_{\mathrm{T}, 0}^{2}\right| / 2 \sigma^{2}\right) /\left(2 \pi \sigma^{2}\right),
$$

with $\sigma^{2}=q_{s}^{2} / 2$ independent of the parton flavor and $x$, with a constant value $q_{s}=0.5 \mathrm{GeV}$. Also, the evolution kernels $\mathcal{K}_{b a}$ in Eq. (2) are taken to include no non-perturbative component. In principle, non-perturbative contributions to Sudakov form factors could be introduced in the $\mathcal{K}_{b a}$ 
kernels of the PB method, and parameterized in terms of non-perturbative functions to be fitted to experimental data (similarly to what is done in other approaches, e.g. [10-13]). For simplicity, however, at present we take the kernels $\mathcal{K}_{b a}$ to be purely perturbative.

Numerical solutions to Eq. (2) at NLO have been used, along with NLO calculations of Drell-Yan (DY) production in the MADGrAPH5_AMC@NLO [14] framework, to compute vectorboson transverse momentum spectra at LHC energies [15] and fixed-target energies [16], and to extract TMD densities from fits [9] to precision deep inelastic scattering (DIS) HERA data. Once the evolution scale is specified in terms of kinematic variables, the PB method allows the explicit calculation of the kinematics at every branching vertex. In this approach, once the TMD distribution $\widetilde{A}_{a}\left(x, \boldsymbol{k}, \mu^{2}\right)$ evaluated at the scale $\mu^{2}$ is known, the corresponding TMD parton shower can be generated by backward evolution.

\section{Jet final states}

As an example of the successful matching of the MC@NLO calculations with the PB evolution of the TMD, in Figs. 1 we compute the azimuthal separation $\Delta \phi_{12}$ of the dijet system containing b-hadrons (b-jets), for dijet mass $m_{j j}>110 \mathrm{GeV}$. The result is compared with the experimental measurement by ATLAS [17] at $\sqrt{s}=7 \mathrm{TeV}$. In the calculation we use MAdGraph5_AMC@NLO [14] to generate $2 \rightarrow 2 \mathrm{QCD}$ events at NLO in $p p$ collisions at a center-of-mass energy $\sqrt{s}=7 \mathrm{TeV}$. The $\mathrm{PB}$ evolution is applied using the generator CASCADE [18]. We use this to generate the PB shower, and for all calculations we apply the PB parton distributions obtained from DIS fits in [9] with $\alpha_{S}\left(M_{Z}\right)=0.118$. The PB-MC@NLO prediction provides a very good description of the whole $\Delta \phi_{12}$ spectrum.

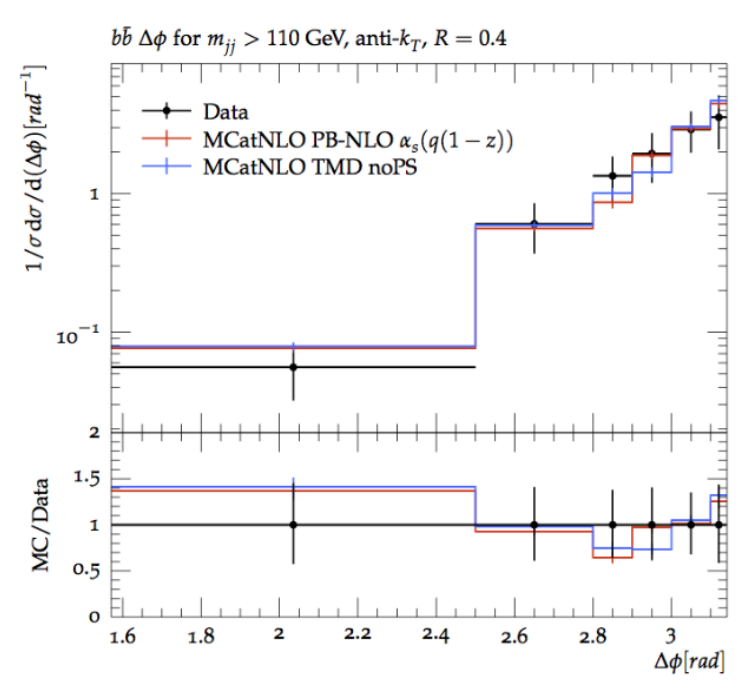

Figure 1: Azimuthal separation $\Delta \phi_{12}$ of the dijet system containing b-jets. Experimental measurement by ATLAS [17] at $\sqrt{s}=7 \mathrm{TeV}$ is compared with results from the PB-MC@NLO calculation.

There are however other jet observables for which the NLO correction is not sufficient for a good description of the data. In Fig. 2 we consider the jet multiplicity associated with DY events. 
The calculation corresponds to $Z+1$ jet at NLO using PB+MC@NLO. The results are compared with ATLAS measurements [19].

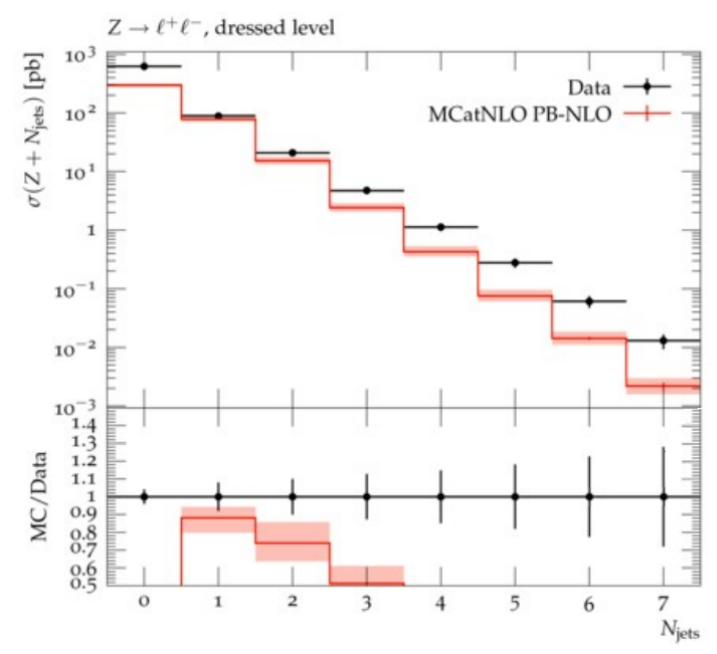

Figure 2: Exclusive jet multiplicity in the production of a $Z$-boson in association with jets. Experimental measurement by ATLAS [19] at $\sqrt{s}=13 \mathrm{TeV}$ is compared with results from the PB+MC@NLO calculation.

Only the 1-jet multiplicity, for which the calculation is NLO accurate, is well described in Fig. 2. The LO accurate 2-jet multiplicity is not well described and the difference between the data and the prediction worsens as we go further in multiplicity. This behaviour indicates that in order to have a good description of multiple hard, well separated jets while preserving the very good description of observables sensitive to resummation effects we need a suitable merging procedure. This merging algorithm needs to be compatible with the use of TMD and corresponding TMD evolution.

In addition, in Fig. 3 we calculate the transverse momentum spectrum of DY lepton pairs from $Z$-boson decay at $8 \mathrm{TeV}$. The event selection follows the analysis performed by ATLAS [20]. The analysis is performed using Rivet [21]. The predictions are computed using $Z+0$ as well as $Z+1$ matrix elements at LO. In Fig. 3 (right) the $Z+0$ calculation is done off-shell using the KATIE generator [22] based on high-energy factorization [23, 24].

We observe in Fig. 3 that the off-shellness of the ME only plays a role at high $p_{T}$ of the lepton pair. Additionally Fig. 3 shows that the $Z+1$ jet contribution has a large effect at high $p_{T}$ of the lepton pair while the $Z+0$ jet calculation is significant at small $p_{T}$. The transition region where the two calculations overlap lies around $20 \mathrm{GeV}$. A suitable merging algorithm should be able to achieve a smooth transition between the $Z+0$ and $Z+1$ jet contributions by enforcing the $Z+0$ jet contribution to be exclusive, suppressing doubly counted events in the overlapping region.

\section{Conclusion}

While the PB+MC@NLO calculations have been successful in describing several observables in high-energy hadronic collisions we have shown that some observables point to the necessity of higher order corrections to describe multi-jet dynamics in PB calculations. We have indicated that 

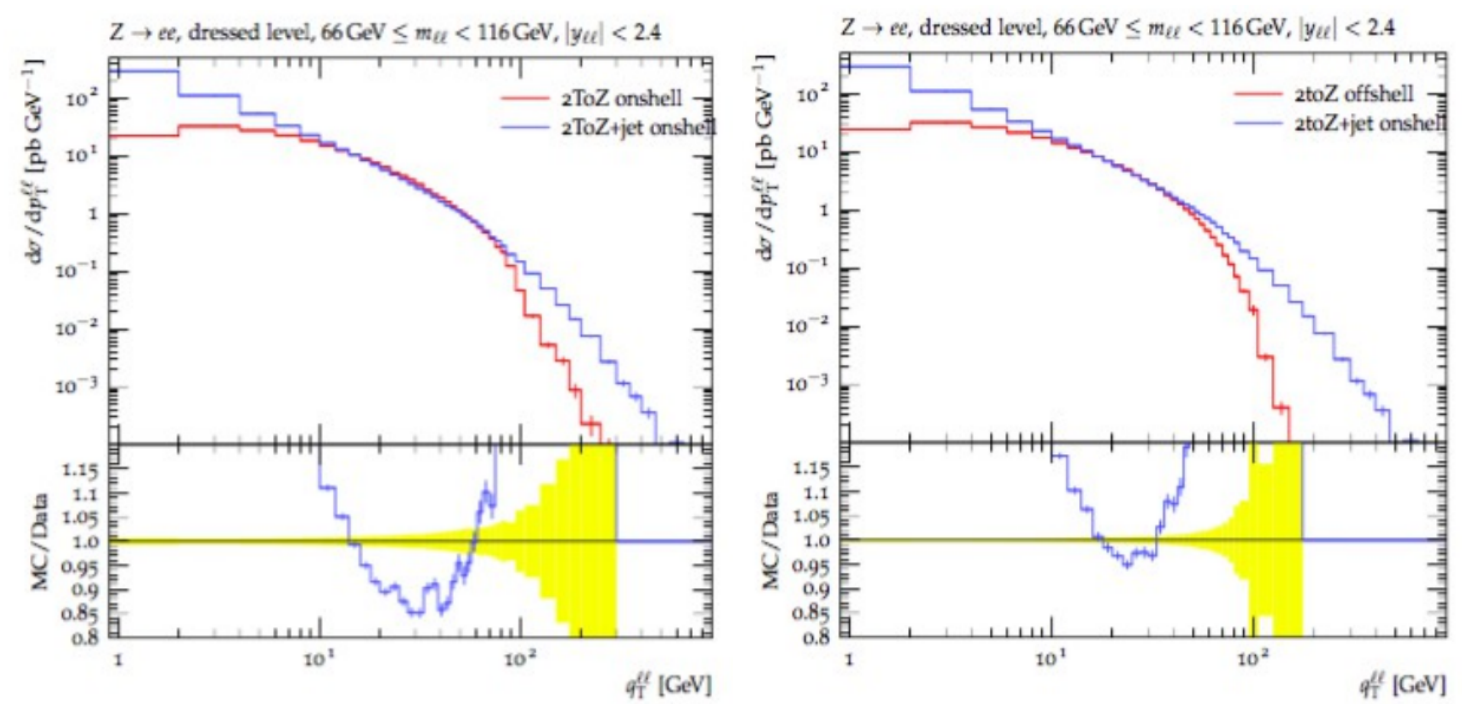

Figure 3: Transverse momentum $p_{T}$ spectrum of $Z$-bosons DY lepton pairs from $Z$-boson decays at $8 \mathrm{TeV}$. The event selection follows the analysis performed by ATLAS [20]. The $Z+0$ and $Z+1$ jet calculations are shown, where the $Z+0$ is computed on-shell (left) as well as off-shell (right).

a new merging algorithm is needed that incorporates the physics of initial-state TMD evolution in multi-jet computations. This new merging algorithm should also allow to include off-shell matrix element in combination with the TMD.

\section{References}

[1] R. Angeles-Martinez et al., Acta Phys. Polon. B 46 (2015) 2501 [arXiv:1507.05267 [hep-ph]].

[2] F. Hautmann, H. Jung, A. Lelek, V. Radescu and R. Zlebcik, JHEP 1801 (2018) 070 [arXiv:1708.03279 [hep-ph]].

[3] B. R. Webber, Ann. Rev. Nucl. Part. Sci. 36 (1986) 253.

[4] R. K. Ellis, W. J. Stirling and B. R. Webber, QCD and collider physics, CUP 1996.

[5] F. Hautmann, H. Jung, A. Lelek, V. Radescu and R. Zlebcik, Phys. Lett. B 772 (2017) 446 [arXiv:1704.01757 [hep-ph]].

[6] G. Marchesini and B. R. Webber, Nucl. Phys. B 310 (1988) 461.

[7] S. Catani, G. Marchesini and B. R. Webber, Nucl. Phys. B 349 (1991) 635.

[8] F. Hautmann, L. Keersmaekers, A. Lelek and A. M. Van Kampen, Nucl. Phys. B 949 (2019) 114795 [arXiv:1908.08524].

[9] A. Bermudez Martinez et al., Phys. Rev. D 99 (2019) 074008 [arXiv:1804.11152 [hep-ph]].

[10] G. Ladinsky and C. Yuan, Phys.Rev. D50 (1994) 4239 [hep-ph/9311341]. 
[11] C. Balazs and C. P. Yuan, Phys. Rev. D56 (1997) 5558 [hep-ph/9704258].

[12] F. Landry, R. Brock, P. M. Nadolsky and C. P. Yuan, Phys. Rev. D67 (2003) 073016 [hep-ph/0212159].

[13] P. Nadolsky et al., "The qt resummation portal.” http://hep.pa.msu. edu/resum/.

[14] J. Alwall et al., JHEP 1407 (2014) 079 [arXiv:1405.0301 [hep-ph]].

[15] A. Bermudez Martinez et al., Phys. Rev. D 100 (2019) 074027 [arXiv:1906.00919 [hep-ph]].

[16] A. Bermudez Martinez et al., Eur. Phys. J. C 80 (2020) 598 [arXiv:2001.06488 [hep-ph]].

[17] G. Aad et al. [ATLAS Collaboration], Eur. Phys. J. C 71 (2011) 1846 [arXiv:1109.6833 [hep-ex]].

[18] H. Jung et al., in preparation; Eur. Phys. J. C 70 (2010) 1237 [arXiv:1008.0152 [hep-ph]].

[19] M. Aaboud et al. [ATLAS Collaboration], Eur. Phys. J. C 77 (2017) 361 [arXiv:1702.05725 [hep-ex]].

[20] G. Aad et al. [ATLAS Collaboration], Eur. Phys. J. C 76 (2016) 291 [arXiv:1512.02192 [hep-ex]].

[21] A. Buckley et al., Comput. Phys. Commun. 184 (2013) 2803 [arXiv:1003.0694 [hep-ph]].

[22] A. van Hameren, Comput. Phys. Commun. 224 (2018) 371 [arXiv:1611.00680 [hep-ph]].

[23] S. Catani, M. Ciafaloni and F. Hautmann, Phys. Lett. B 242 (1990) 97.

[24] S. Catani, M. Ciafaloni and F. Hautmann, Nucl. Phys. B 366 (1991) 135. 